\title{
Livestock research for rural development - a computerized journal
}

\author{
A. W. Speedy ${ }^{1}$ and T. R. Preston ${ }^{2}$ \\ ${ }^{1}$ Department of Plant Sciences, University of Oxford, Oxford OX1 3RB \\ ${ }^{2}$ Convenio Interinstitucional par la Producción Agropecuaria del Valle del Rio Cauca, (CIPAV), AA7482, Cali, Colombia
}

\section{Introduction}

The provision of appropriate information and establishment of effective communication is one of the most effective contributions that can be made to the development of sustainable systems of agriculture and forestry in developing countries.

The development of new livestock, agroforestry and integrated farm technology based on local resources in Latin America, the Caribbean, Africa and Asia is advancing on the basis of work carried out in those countries. There was a need for a cheap and effective communication medium for publication of research and exchange of information in the field. Conventional journals are expensive, too slow in publication and generally unavailable to many workers in developing countries. Computers, however, have become widespread and, with reducing costs, have become an integrated part of development projects.

Livestock Research for Rural Development was started in 1989 as a medium for the publication of research on animal production based on locally available resources in developing countries. The majority of authors are young research workers from those countries. There have now been five issues ( 50 papers) and there are over 1500 recipients.

\section{Paper presentation}

Papers are submitted in WordPerfect (or other standard wordprocessor) format or ASCII text files on disk. Figures may now also be submitted in HARVARD GRAPHICS. CHT format. The papers are distributed together with a simple but efficient textreader program which allows selection from the contents page, scrolling through the paper on-screen and printing to a standard (Epsom-compatible) printer. The disk is readable on any IBM-compatible computer, with a minimum of $512 \mathrm{~K}$ RAM. The user requires no manual; all instructions are shown onscreen and the program uses simple menus and cursor movements.

\section{Paper distribution}

Distribution has been organized through a network of cooperators throughout the world and, to date, subscribers have received a copy by exchanging disks (avoiding currency exchange problems). The majority receive copies through the support of the Centre for Technical Cooperation in Agriculture (CTA), the International Foundation for Science (IFS) and the Food and Agriculture Organization of the United Nations (FAO). Scientists and institutions in developed countries must pay a modest subscription.

With the distribution of disks to members in African, Caribbean and Pacific (ACP) countries, a questionnaire was circulated by CTA. Forty-three letters were forwarded with answers to five questions. Thirty-three out of the 43 respondents (77\%) had access to computers and indicated their desire both to continue to receive and to submit papers to the journal.

The journal may also be distributed to institutes and universities by electronic mail, using data compression and encoding. As communications improve, it is possible that workers in developing countries can start to use the new methods. This will replace the printed book and journal as the main means of information transfer in the world. It offers major possibilities of better research and more appropriate development. 